Article

\title{
Metabolic Analysis of Various Date Palm Fruit (Phoenix dactylifera L.) Cultivars from Saudi Arabia to Assess Their Nutritional Quality
}

\section{Ismail Hamad ${ }^{1,6, \dagger}$, Hamada AbdElgawad ${ }^{2,3, \dagger}$, Soad Al Jaouni ${ }^{4}$, Gaurav Zinta ${ }^{2}$, Han Asard ${ }^{2}$, Sherif Hassan ${ }^{1,3}$, Momtaz Hegab ${ }^{3}$, Nashwa Hagagy 5 and Samy Selim ${ }^{1,5, *}$}

1 Department of Clinical Laboratory Sciences, College of Applied Medical Sciences, Aljouf University, Sakaka 2014, Saudi Arabia; E-Mails: ismailhamad@yahoo.com (I.H.); abood127@yahoo.com (S.H.)

2 Laboratory for Molecular Plant Physiology and Biotechnology, Department of Biology, University of Antwerp, Groenenborgerlaan 171, B-2020, Antwerp 2020, Belgium;

E-Mails: hamada.abdelgawad@uantwerpen.be (H.A.); gaurav.zinta@uantwerpen.be (G.Z.); han.asard@uantwerpen.be (H.A.)

3 Department of Botany, Faculty of Science, University of Beni-Suef, Beni-Suef 62511, Egypt; E-Mail: momtazyehya@hotmail.com

4 YAJ Prophatic Medicine Application, College of Medicine, King Abdulaziz University, P.O. Box 80215, Jeddah 21589, Saudi Arabia; E-Mail: saljaouni@kau.edu.sa

5 Microbiology and Botany Department, Faculty of Science, Suez Canal University, Ismailia 41522 , Egypt; E-Mail: nashwa_hagag@hotmail.com

6 Biochemistry Department, Bahri University, Khartoum 1660, Sudan

$\dagger$ These authors contributed equally to this work.

* Author to whom correspondence should be addressed; E-Mail: sabdulsalam@ju.edu.sa; Tel./Fax: +966-535-268-405.

Academic Editor: Marcello Iriti

Received: 21 June 2015 / Accepted: 16 July 2015 / Published: 27 July 2015

\begin{abstract}
Date palm is an important crop, especially in the hot-arid regions of the world. Date palm fruits have high nutritional and therapeutic value and possess significant antibacterial and antifungal properties. In this study, we performed bioactivity analyses and metabolic profiling of date fruits of 12 cultivars from Saudi Arabia to assess their nutritional value. Our results showed that the date extracts from different cultivars have different free radical
\end{abstract}


scavenging and anti-lipid peroxidation activities. Moreover, the cultivars showed significant differences in their chemical composition, e.g., the phenolic content (10.4-22.1 mg/100 g DW), amino acids (37-108 $\mu \mathrm{mol} \cdot \mathrm{g}^{-1} \mathrm{FW}$ ) and minerals (237-969 $\left.\mathrm{mg} / 100 \mathrm{~g} \mathrm{DW}\right)$. Principal component analysis (PCA) showed a clear separation of the cultivars into four different groups. The first group consisted of the Sokary, Nabtit Ali cultivars, the second group of Khlas Al Kharj, Khla Al Qassim, Mabroom, Khlas Al Ahsa, the third group of Khals Elshiokh, Nabot Saif, Khodry, and the fourth group consisted of Ajwa Al Madinah, Saffawy, Rashodia, cultivars. Hierarchical cluster analysis (HCA) revealed clustering of date cultivars into two groups. The first cluster consisted of the Sokary, Rashodia and Nabtit Ali cultivars, and the second cluster contained all the other tested cultivars. These results indicate that date fruits have high nutritive value, and different cultivars have different chemical composition.

Keywords: date palm; lipid peroxidation; antioxidants; antimicrobial; minerals; phenolics; amino acids; organic acids; metabolomics

\section{Introduction}

Date palm (Phoenix dactylifera L.) is the most successful and commercially important crop in the hot-arid regions of the world, e.g., Saudi Arabia, Emirates and Egypt [1,2]. In these countries, date palm products are commonly used for human and animal consumption, pharmaceuticals, cosmetics, carpentry, and firewood. A large number of date palm cultivars are known, however, until now only a few of these cultivars have been evaluated for chemical composition and nutritional quality [1,2].

Date fruits have a great importance from both a nutritional and therapeutic point of view [3,4]. They are rich sources of sugars, vitamins, minerals and fibers. In some date varieties, the sugar content of the fruits reaches up to $88 \%$, and such fruits are considered a high-energy food source [5]. Moreover, date fruits possess antioxidant and antimutagenic properties [6,7], attributable to their high levels of polyphenolic compounds and vitamins [7,8]. For example, Al-Farsi et al. [9] found that total phenolic content ranged from 172 to $246 \mathrm{mg}$ of gallic acid/100 $\mathrm{g}$ in three date varieties grown in Oman. Yousif et al. [10] observed that date fruits have high vitamin levels, e.g., ascorbic acid (2.4-17.5 mg/100 g), thiamine $(0.08-0.13 \mathrm{mg} / 100 \mathrm{~g})$ and riboflavin $(0.13-17.5 \mathrm{mg} / 100 \mathrm{~g})$. Moreover, dates are rich in dietary fiber $(6.4 \%-11.5 \%)$, which further improves their nutritional value and therapeutic utility $[5,11]$. Extracts of dates also show antibacterial and antifungal properties [12-14]. Considering the nutritional importance of dates, studying their biochemical composition and nutritional quality is increasingly being recognized as a worthy and important task.

Varying growth conditions cause changes in the primary and secondary metabolism of plants [15-22]. Similarly, the growth conditions of different regions induce several external and internal changes in the dates. Such changes are often classified on the basis of observed differences in the color and chemical composition of date fruits. For instance, the nutritional quality of dates varies among varieties grown in Algeria [7], Egypt [23], Oman [24,25] and Bahrain [26]. The study of Farag et al. [23] recorded a high variation in the chemical metabolites of 21 Egyptian date varieties. In particular their study revealed that the phenolic contents varied considerably among these varieties $\left[2.3-19 \mathrm{~g} \cdot \mathrm{kg}^{-1} \mathrm{DW}\right]$. Moreover, cluster 
analyses indicated that flavonols and sugars both contribute the most to variety classification. Recently, metabolomics have been successfully applied to investigate the chemical composition of dates to assess their nutritional quality [27-29], and such approaches are crucial to establish links between plant genotypes and phenotypes.

The aim of this study was to evaluate the biological activity and nutritional quality of 12 date varieties from different geographical locations in Saudi Arabia. These varieties correspond to the commonly used ones in this region. In this study, we used high performance liquid chromatography (HPLC) coupled to electrochemical and diode array detection and mass spectrometry (HPLC/PDA/MS). These techniques enabled us to analyze a wide range of metabolites including sugars, amino acids, fatty acids, organic acids, phenolics and flavonoids, and antioxidants in the 12 different palm varieties. We also assessed the macroand microelemental mineral profile and hydrophilic and lipophilic antioxidant contents of these cultivars. To acquire statistical correlations among all the measured parameters and different palm cultivars, we performed principle component analysis (PCA) and hierarchical clustering analysis (HCA).

\section{Results and Discussion}

\subsection{Metabolites}

\subsubsection{Antioxidants}

Glutathione (GSH) and ascorbic acid (ASC) are aqueous phase antioxidants, while tocopherol is lipophilic in nature. GSH content showed a variation among the cultivars, ranging from 0.011 to $0.295 \mu \mathrm{mol} \cdot \mathrm{g}^{-1} \mathrm{FW}$ (Figure 1, Table 1). The highest GSH content was observed for the Rashodia, Khlas Al Ahsa and Nabtit Ali cultivars $\left(0.247,0.177\right.$ and $0.295 \mu \mathrm{mol} \cdot \mathrm{g}^{-1} \mathrm{FW}$, respectively) while Khodry had the lowest GSH content $\left(0.011 \mu \mathrm{mol} \cdot \mathrm{g}^{-1} \mathrm{FW}\right)$. Similarly, ASC content varied significantly among the date cultivars and was in the range of 0.051 and $0.541 \mu \mathrm{mol} \cdot \mathrm{g}^{-1} \mathrm{FW}$ (Table 1). Rashodia, Sokary and Nabtit Ali showed the highest ASC content $\left(0.541,0.526\right.$ and $0.516 \mu \mathrm{mol} \cdot \mathrm{g}^{-1} \mathrm{FW}$ respectively) and Ajwa Al Madinah showed the lowest ASC content $\left(0.051 \mu \mathrm{mol} \cdot \mathrm{g}^{-1} \mathrm{FW}\right)$ (Table 1). Total tocopherol content was in the range of 0.09 to $0.28 \mu \mathrm{mol} \cdot \mathrm{g}^{-1} \mathrm{FW}$ (Table 1 ) where Sokary had the highest content $\left(0.28 \mu \mathrm{mol} \cdot \mathrm{g}^{-1} \mathrm{FW}\right.$ ), and $\mathrm{Khla}$ Al Qassim had the lowest tocopherol content $\left(0.09 \mu \mathrm{mol} \cdot \mathrm{g}^{-1} \mathrm{FW}\right)$ (Table 1). The redox ratios of GSH and ASC, and $\alpha-, \beta$-, $\gamma$ - and $\delta$-tocopherols are listed in Table 1.

\subsubsection{Amino Acids}

Many amino acids were detected in the fruits of the twelve studied cultivars, which were rich in amino acids. Moreover, the cultivars showed significant differences in their amino acids contents (37-108 $\mu \mathrm{mol} \cdot \mathrm{g}^{-1} \mathrm{FW}$ ). Proline was the major amino acid, and it was highly abundant in the Nabitit Ali and Rashodia cultivars ( 85 and $126 \mu \mathrm{mol} \cdot \mathrm{g}^{-1} \mathrm{FW}$, respectively). On the other hand, cysteine was the minor amino acid (0.001-0.11 $\left.\mu \mathrm{mol} \cdot \mathrm{g}^{-1} \mathrm{FW}\right)$ (Table 2). 
Table 1. Concentrations of glutathione (GSH), ascorbate (ASC) and tocopherols (toco), and redox status of GSH and ASC in 12 Saudi date cultivars.

\begin{tabular}{|c|c|c|c|c|c|c|c|c|}
\hline Cultivars & $\begin{array}{c}\text { GSH } \\
\left(\mu \mathrm{mol} \cdot \mathrm{g}^{-1} \mathrm{FW}\right)\end{array}$ & $\begin{array}{c}\text { GSH Redox Status } \\
(\%)\end{array}$ & $\begin{array}{c}\text { ASC } \\
\left(\mu \mathrm{mol} \cdot \mathrm{g}^{-1} \mathrm{FW}\right)\end{array}$ & $\begin{array}{c}\text { ASC Redox Status } \\
(\%)\end{array}$ & $\begin{array}{c}\text { Alfa Toc. } \\
\text { (ng/100 g FW) }\end{array}$ & $\begin{array}{c}\text { Beta Toc. } \\
(\mathrm{ng} / 100 \mathrm{~g} \mathrm{FW})\end{array}$ & $\begin{array}{l}\text { Gamma Toc. } \\
(\mathrm{ng} / 100 \text { g FW) }\end{array}$ & $\begin{array}{c}\text { Delta Toc. } \\
\text { (ng/100 g FW) }\end{array}$ \\
\hline Nabot Saif & $0.025 \pm 0.003$ & $77.366 \pm 5.532$ & $0.254 \pm 0.023$ & $99.259 \pm 2.637$ & $0.086 \pm 0.009$ & $0.023 \pm 0.002$ & $0.016 \pm 0.001$ & $0 \pm 0.0$ \\
\hline Rashodia & $0.247 \pm 0.026$ & $43.523 \pm 5.111$ & $0.541 \pm 0.049$ & $99.643 \pm 2.646$ & $0.124 \pm 0.013$ & $0.013 \pm 0.002$ & $0.021 \pm 0.002$ & $0 \pm 0.0$ \\
\hline Ajwa Al Madinah & $0.062 \pm 0.007$ & $86.049 \pm 5.794$ & $0.051 \pm 0.005$ & $85.244 \pm 3.061$ & $0.212 \pm 0.022$ & $0.022 \pm 0.002$ & $0.022 \pm 0.003$ & $0.003 \pm 0.00$ \\
\hline Khodry & $0.011 \pm 0.001$ & $39.373 \pm 4.728$ & $0.387 \pm 0.035$ & $98.891 \pm 3.551$ & $0.197 \pm 0.02$ & $0.033 \pm 0.002$ & $0.044 \pm 0.004$ & $0.002 \pm 0.00$ \\
\hline Khlas Al Ahsa & $0.177 \pm 0.018$ & $96.507 \pm 22.175$ & $0.206 \pm 0.019$ & $100.791 \pm 3.619$ & $0.14 \pm 0.014$ & $0.026 \pm 0.002$ & $0.016 \pm 0.001$ & $0.010 \pm 0.001$ \\
\hline Sokary & $0.059 \pm 0.006$ & $21.736 \pm 0.580$ & $0.526 \pm 0.047$ & $87.570 \pm 2.326$ & $0.218 \pm 0.022$ & $0.019 \pm 0.0021$ & $0.043 \pm 0.005$ & $0.011 \pm 0.001$ \\
\hline Saffawy & $0.039 \pm 0.005$ & $48.128 \pm 5.652$ & $0.423 \pm 0.038$ & $98.662 \pm 2.621$ & $0.179 \pm 0.0188$ & $0.023 \pm 0.0024$ & $0.038 \pm 0.0$ & $0.04 \pm 0.002$ \\
\hline Khlas Al Kharj & $0.054 \pm 0.006$ & $94.605 \pm 11.110$ & $0.346 \pm 0.031$ & $100.261 \pm 2.717$ & $0.113 \pm 0.0177$ & $0.027 \pm 0.0024$ & $0.018 \pm 0.001$ & $0.003 \pm 0.0$ \\
\hline Mabroom & $0027 \pm 0.003$ & $38.279 \pm 1.021$ & $0.296 \pm 0.027$ & $99.577 \pm 14.785$ & $0.197 \pm 0.02$ & $0.014 \pm 0.0018$ & $0.023 \pm 0.002$ & $0.008 \pm 0.00$ \\
\hline Khla Al Qassim & $0.039 \pm 0.004$ & $72.546 \pm 1.935$ & $0.421 \pm 0.038$ & $99.408 \pm 14.760$ & $0.072 \pm 0.007$ & $0.025 \pm 0.001$ & $0.0157 \pm 0.003$ & $0.001 \pm 0.00$ \\
\hline Nabtit AIi & $0.295 \pm 0.031$ & $47.527 \pm 1.267$ & $0.516 \pm 0.049$ & $86.407 \pm 6.863$ & $0.158 \pm 0.0165$ & $0.020 \pm 0.0012$ & $0.038 \pm 0.004$ & $0 \pm 0.00$ \\
\hline Khals El Shiokh & $0.065 \pm 0.007$ & $82.268 \pm 2.194$ & $0.376 \pm 0.034$ & $100.600 \pm 15.382$ & $0.163 \pm 0.017$ & $0.019 \pm 0.0021$ & $0.016 \pm 0.002$ & $0.005 \pm 0.00$ \\
\hline$p$ value & 0 & 0 & 0 & 0.093 & 0 & 0.154 & 0 & 0 \\
\hline
\end{tabular}


Table 2. Concentrations of amino acids $\left(\mu \mathrm{mol} \cdot \mathrm{g}^{-1} \mathrm{FW}\right)$ in 12 Saudi date cultivars.

\begin{tabular}{|c|c|c|c|c|c|c|c|c|c|c|}
\hline Cultivars & Proline & Glycine & Lysine & Histidine & Alanine & Arginie & Ornithine & Glutamine & Asparagine & Isoleucine \\
\hline Nabot Saif & $76 \pm 11$ & $78 \pm 8.8$ & $3.8 \pm 0.6$ & $1.16 \pm 0.18$ & $19.2 \pm 2.1$ & $0.43 \pm 0.0$ & $0.03 \pm 0.00$ & $0.41 \pm 0.04$ & $0.72 \pm 0.07$ & $0.10 \pm 0.01$ \\
\hline Rashodia & $85 \pm 13$ & $39 \pm 4.5$ & $2.9 \pm 0.4$ & $0.84 \pm 0.13$ & $11.3 \pm 1.2$ & $2.7 \pm 0.3$ & $0.13 \pm 0.01$ & $1.61 \pm 0.16$ & $1.101 \pm 0.1$ & $0.15 \pm 0.01$ \\
\hline Ajwa Al Madinah & $16 \pm 2.6$ & $65 \pm 7.4$ & $7.3 \pm 1.1$ & $0.99 \pm 0.1$ & $9.2 \pm 1.0$ & $1.42 \pm 0.1$ & $0.15 \pm 0.01$ & $1.02 \pm 0.1$ & $0.26 \pm 0.03$ & $0.15 \pm 0.01$ \\
\hline Khodry & $11 \pm 1.7$ & $57 \pm 6.5$ & $3.2 \pm 0.5$ & $0.98 \pm 0.1$ & $8.07 \pm 0.9$ & $0.31 \pm 0.0$ & $0.042 \pm 0.0$ & $0.14 \pm 0.01$ & $1.07 \pm 0.1$ & $0.09 \pm 0.0$ \\
\hline Khlas Al Ahsa & $14 \pm 2.2$ & $75 \pm 8.5$ & $4.4 \pm 0.6$ & $1.47 \pm 0.2$ & $12.8 \pm 1.4$ & $0.24 \pm 0.0$ & $0.13 \pm 0.02$ & $0.56 \pm 0.05$ & $0.518 \pm 0.2$ & $0.15 \pm 0.05$ \\
\hline Sokary & $12 \pm 19$ & $13 \pm 1.5$ & $2.2 \pm 0.3$ & $1.40 \pm 0.2$ & $5.8 \pm 0.64$ & $1.11 \pm 0.1$ & $0.1 \pm 0.01$ & $1.20 \pm 0.1$ & $4.4 \pm 0.4$ & $1.79 \pm 0.17$ \\
\hline Saffawy & $28 \pm 4.3$ & $49 \pm 5.5$ & $3.2 \pm 0.5$ & $0.97 \pm 0.1$ & $11.5 \pm 1.2$ & $0.30 \pm 0.0$ & $0.038 \pm 0.0$ & $0.30 \pm 0.03$ & $1.2 \pm 0.1$ & $0.08 \pm 0.00$ \\
\hline Khlas Al Kharj & $8 \pm 1.3$ & $49 \pm 5.6$ & $3.4 \pm 0.5$ & $1.20 \pm 0.2$ & $7.6 \pm 0.8$ & $0.43 \pm 0.0$ & $0.1 \pm 0.02$ & $0.30 \pm 0.03$ & $0.07 \pm 0.0$ & $0.13 \pm 0.01$ \\
\hline Mabroom & $10 \pm 1.5$ & $57 \pm 6.5$ & $4.52 \pm 0$ & $0.07 \pm 0.01$ & $13.6 \pm 1.5$ & $0.21 \pm 0.0$ & $0.021 \pm 0.0$ & $0.43 \pm 0.01$ & $0.93 \pm 0.08$ & $0.081 \pm 0.0$ \\
\hline Khla Al Qassim & $9.5 \pm 1.5$ & $47 \pm 5.3$ & $1.9 \pm 0.3$ & $0.91 \pm 0.14$ & $16 \pm 1.8$ & $0.99 \pm 0.1$ & $0.06 \pm 0.01$ & $0.25 \pm 0.02$ & $0.09 \pm 0.0$ & $0.11 \pm 0.01$ \\
\hline Nabtit AIi & $126 \pm 6$ & $17 \pm 1.9$ & $1.0 \pm 0.0$ & $0.97 \pm 0.15$ & $7.07 \pm 0.7$ & $2.65 \pm 0.2$ & $0.13 \pm 0.0$ & $3.5 \pm 0.34$ & $1.4 \pm 0.15$ & $1.39 \pm 0.14$ \\
\hline Khals El Shiokh & $10.3 \pm 1.6$ & $38 \pm 4.3$ & $3.0 \pm 0.4$ & $1.09 \pm 0.17$ & $13.2 \pm 1.5$ & $0.50 \pm 0.0$ & $0.09 \pm 0.01$ & $0.48 \pm 005$ & $0.97 \pm 0.1$ & $0.15 \pm 0.01$ \\
\hline$p$ value & 0.0 & 0.0 & 0.0 & 0.0 & 0.0 & 0.0 & 0.0 & 0.0 & 0.0 & 0.0 \\
\hline Cultivars & Leucine & Methionine & Threonine & Valine & Serine & Phenylalanine & Glutamic acid & Cysteine & Tyrosine & \\
\hline Nabot Saif & $0.014 \pm 0.0$ & $0.012 \pm 0.00$ & $0.074 \pm 0.0$ & $1.157 \pm 0.2$ & $0.13 \pm 0.0$ & $0.38 \pm 0.05$ & $1.0 \pm 0.18$ & $0.01 \pm 0.0$ & $0.462 \pm 0.05$ & \\
\hline Rashodia & $0.018 \pm 0.0$ & $0.016 \pm 0.0$ & $0.112 \pm 0.01$ & $0.93 \pm 0.17$ & $0.19 \pm 0.0$ & $0.19 \pm 0.03$ & $0.7 \pm 0.13$ & $0.02 \pm 0.0$ & $0.39 \pm 0.04$ & \\
\hline Ajwa Al Madinah & $0.02 \pm 0.00$ & $0.021 \pm 0.00$ & $0.027 \pm 0.0$ & $3.13 \pm 0.6$ & $0.19 \pm 0.0$ & $0.99 \pm 0.14$ & $0.8 \pm 0.15$ & $0.001 \pm 0.0$ & $0.80 \pm 0.08$ & \\
\hline Khodry & $0.1 \pm 0.01$ & $0.09 \pm 0.01$ & $0.110 \pm 0.01$ & $1.188 \pm 0.2$ & $0.11 \pm 0.0$ & $0.36 \pm 0.05$ & $0.8 \pm 0.15$ & $0.009 \pm 0.0$ & $0.35 \pm 0.00$ & \\
\hline Khlas Al Ahsa & $0.06 \pm 0.00$ & $0.05 \pm 0.00$ & $0.053 \pm 0.00$ & $0.80 \pm 0.15$ & $0.19 \pm 0.0$ & $0.70 \pm 0.1$ & $1.3 \pm 0.22$ & $0.001 \pm 0.0$ & $0.94 \pm 0.1$ & \\
\hline Sokary & $0.19 \pm 0.02$ & $0.173 \pm 0.02$ & $0.45 \pm 0.05$ & $0.493 \pm 0.09$ & $2.20 \pm 0.2$ & $0.43 \pm 0.07$ & $1.2 \pm 0.2$ & $0.16 \pm 0.01$ & $0.74 \pm 0.08$ & \\
\hline Saffawy & $0.07 \pm 0.00$ & $0.067 \pm 0.0$ & $0.12 \pm 0.01$ & $0.71 \pm 0.13$ & $0.11 \pm 0.0$ & $0.11 \pm 0.02$ & $0.8 \pm 0.1$ & $0.007 \pm 0.0$ & $0.06 \pm 0.0$ & \\
\hline Khlas Al Kharj & $0.25 \pm 0.02$ & $0.22 \pm 0.02$ & $0.0074 \pm 0.0$ & $0.87 \pm 0.17$ & $0.17 \pm 0.0$ & $0.32 \pm 0.05$ & $1.0 \pm 0.1$ & $0.007 \pm 0.0$ & $0.63+0.07$ & \\
\hline Mabroom & $0.07 \pm 0.00$ & $0.064 \pm 0.00$ & $0.095 \pm 0.01$ & $0.81 \pm 0.15$ & $0.10 \pm 0.0$ & $0.44 \pm 0.07$ & $0.06 \pm 0.0$ & $0.007 \pm 0.0$ & $0.42 \pm 0.05$ & \\
\hline Khla Al Qassim & $0.082 \pm 0.0$ & $0.072 \pm 0.00$ & $0.009 \pm 0.00$ & $0.47 \pm 0.08$ & $0.13 \pm 0.0$ & $0.18 \pm 0.03$ & $0.79 \pm 0.1$ & $0.15 \pm 0.02$ & $0.43 \pm 0.05$ & \\
\hline Nabtit AIi & $0.084 \pm 0.0$ & $0.074 \pm 0.00$ & $0.15 \pm 0.01$ & $0.69 \pm 0.13$ & $1.72 \pm 0.1$ & $0.12 \pm 0.02$ & $0.85 \pm 0.1$ & $0.11 \pm 0.01$ & $0.48 \pm 0.05$ & \\
\hline Khals El Shiokh & $0.082 \pm 0.0$ & $0.071 \pm 0.01$ & $0.09 \pm 0.01$ & $0.65 \pm 0.1$ & $0.18 \pm 0.0$ & $0.27 \pm 0.04$ & $0.95 \pm 0.17$ & $0.11 \pm 0.01$ & $0.613 \pm 0.07$ & \\
\hline$p$ value & 0.0 & 0.0 & 0.0 & 0.0 & 0.0 & 0.0 & 0.0 & 0.0 & 0.0 & \\
\hline
\end{tabular}




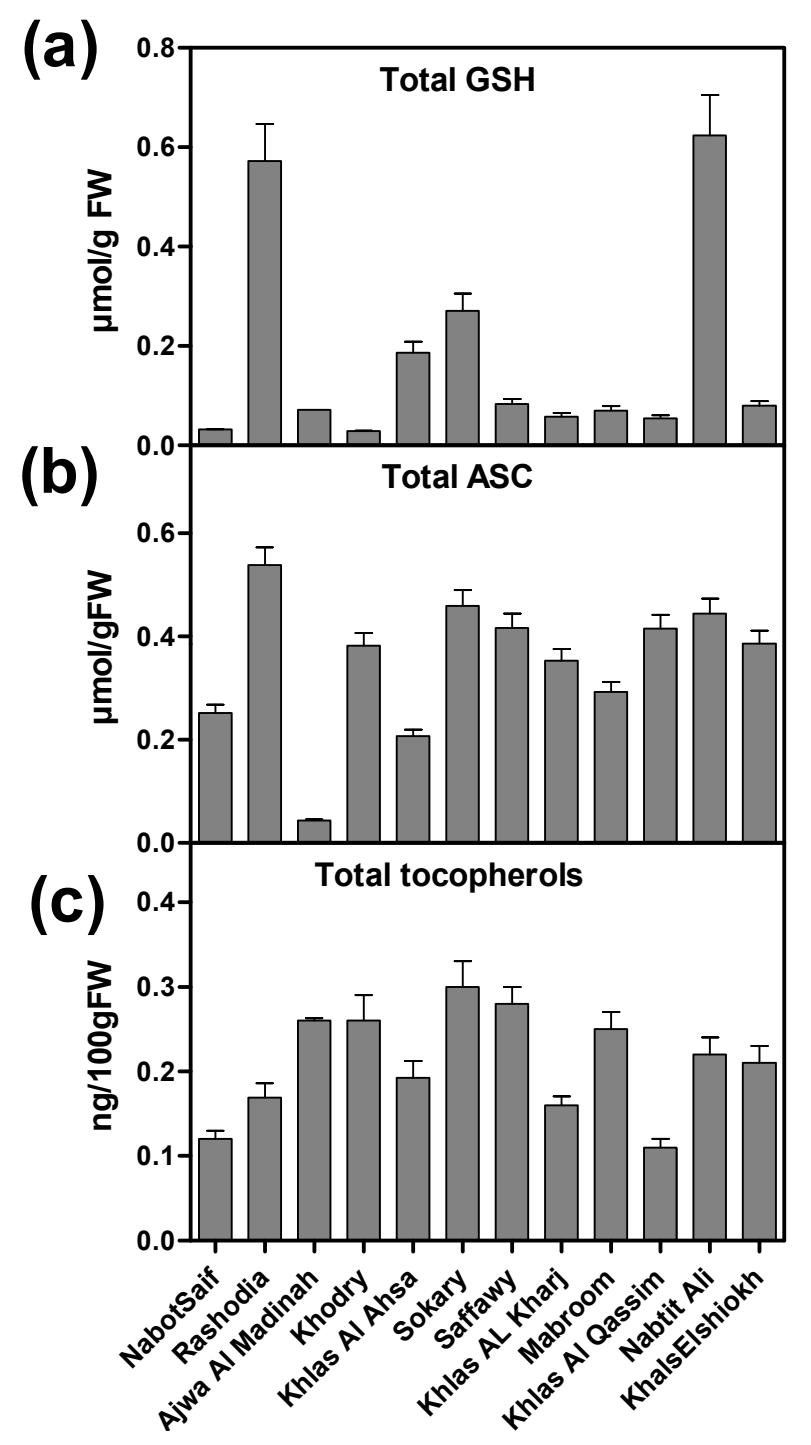

Figure 1. Antioxidant metabolites content: (a) Total glutathione (GSH); (b) total ascorbate (ASC); (c) total tocopherols of 12 Saudi date cultivars.

\subsubsection{Sugars}

We measured monosaccharides (glucose, fructose), disaccharides (sucrose) and total sugar content in the 12 date cultivars (Table 3 ). The total sugar content in the date cultivars was quite high, e.g., Khla Al Qassim had $0.11 \mathrm{mg} / 100 \mathrm{~g} \mathrm{FW}$ and $0.3 \mathrm{mg} / 100 \mathrm{~g} \mathrm{FW}$, suggesting that date fruit are rich sources of sugars. Most of the studied cultivars had higher glucose and fructose concentrations, conversely Nabtit Ali, Sokary and Rashodia cultivars has higher sucrose levels (Table 3).

\subsubsection{Organic Acids}

The average content of total organic acids in dates of tested varieties were 17 to $26 \mathrm{mg} \cdot \mathrm{g}^{-1} \mathrm{FW}$. Six organic acids were identified, among which malic acid was the predominant organic acid, and its concentration ranged from 5 to $10 \mathrm{mg} \cdot \mathrm{g}^{-1} \mathrm{DW}$, followed by lesser amounts of succinic acid, isobutyric acid, citric acid, oxalic acid and formic acid (Table 3 ). 
Table 3. Concentrations of sugars (mg/100 $\mathrm{g} \mathrm{FW})$ and organic acids $\left(\mathrm{mg} \cdot \mathrm{g}^{-1} \mathrm{FW}\right)$ in $12 \mathrm{Saudi}$ date cultivars.

\begin{tabular}{|c|c|c|c|c|c|c|c|c|c|}
\hline Cultivars & Glucose & Fructose & Sucrose & Oxalic & Malic & Succinic & Citric & Isobutyric & Formic \\
\hline Nabot Saif & $50.1 \pm 0.0$ & $58.8 \pm 1.8$ & $26.55 \pm 0.0$ & $1.93 \pm 0.11$ & $9.66 \pm 0.58$ & $1.59 \pm 0.08$ & $2.70 \pm 0.14$ & $2.34 \pm 0.12$ & $0.29 \pm 0.02$ \\
\hline Rashodia & $42.5 \pm 0.6$ & $53.0 \pm 0.0$ & $112.5 \pm 0.0$ & $1.64 \pm 0.1$ & $7.03 \pm 0.79$ & $5.69 \pm 0.7$ & $2.86 \pm 0.38$ & $2.88 \pm 0.15$ & $0.37 \pm 0.02$ \\
\hline Ajwa Al Madinah & $35.4 \pm 0.5$ & $39.4 \pm 2.5$ & $13.45 \pm 0.2$ & $1.46 \pm 0.09$ & $10.12 \pm 1.18$ & $0.76 \pm 0.08$ & $2.01 \pm 0.23$ & $3.12 \pm 0.19$ & $0.35 \pm 0.02$ \\
\hline Khodry & $58.1 \pm 0.0$ & $69.16 \pm 2.1$ & $19.42 \pm 0.0$ & $2.24 \pm 0.13$ & $11.41 \pm 0.69$ & $1.19 \pm 0.07$ & $2.31 \pm 0.22$ & $2.09 \pm 0.11$ & $0.32 \pm 0.02$ \\
\hline Khlas Al Ahsa & $58.2 \pm 3.6$ & $74.1 \pm 4.7$ & $17.9 \pm 0.27$ & $2.73 \pm 0.17$ & $13.98 \pm 0.85$ & $1.23 \pm 0.08$ & $2.33 \pm 0.14$ & $2.01 \pm 0.12$ & $0.17 \pm 0.01$ \\
\hline Sokary & $1.5 \pm 1.8$ & $59.5 \pm 3.7$ & $138.5 \pm 5.0$ & $2.18 \pm 0.13$ & $10.43 \pm 0.55$ & $9.26 \pm 0.56$ & $4.65 \pm 0.25$ & $2.94 \pm 0.18$ & $0.29 \pm 0.02$ \\
\hline Saffawy & $47.3 \pm 0.07$ & $54.26 \pm 2.4$ & $28.7 \pm 1.04$ & $1.82 \pm 0.1$ & $9.10 \pm 0.47$ & $1.86 \pm 0.11$ & $0.95 \pm 0.05$ & $3.23 \pm 0.18$ & $0.21 \pm 0.01$ \\
\hline Khlas Al Kharj & $95.40 \pm 0.0$ & $112.7 \pm 3.4$ & $31.9 \pm 0.0$ & $1.90 \pm 0.1$ & $17.68 \pm 1.08$ & $1.82 \pm 0.09$ & $0.93 \pm 0.05$ & $3.30 \pm 0.17$ & $0.27 \pm 0.02$ \\
\hline Mabroom & $46.30 \pm 0.70$ & $62.0 \pm .00$ & $20.1 \pm 0.0$ & $1.85 \pm 0.1$ & $8.68 \pm 0.88$ & $1.07 \pm 0.13$ & $2.14 \pm 0.26$ & $2.62 \pm 0.31$ & $0.17 \pm 0.02$ \\
\hline Khla Al Qassim & $79.6 \pm 0.0$ & $101.2 \pm 0.0$ & $26.1 \pm 0.0$ & $1.57 \pm 0.08$ & $13.20 \pm 1.76$ & $1.40 \pm 0.16$ & $2.41 \pm 0.27$ & $2.07 \pm 0.23$ & $0.20 \pm 0.03$ \\
\hline Nabtit AIi & $21.08 \pm 0.3$ & $23.20 \pm 1.47$ & $150.5 \pm 2.2$ & $0.83 \pm 0.04$ & $10.01 \pm 1.1$ & $8.66 \pm 0.82$ & $4.43 \pm 0.42$ & $2.16 \pm 0.22$ & $0.23 \pm 0.03$ \\
\hline Khals El Shiokh & $58.2 \pm 0.0$ & $71.29 \pm 2.2$ & $9.23 \pm 0.0$ & $2.49 \pm 0.15$ & $12.94 \pm 0.78$ & $0.62 \pm 0.04$ & $1.98 \pm 0.12$ & $1.70 \pm 0.1$ & $0.19 \pm 0.01$ \\
\hline$p$ value & 0.01 & 0 & 0.05 & 0 & 0 & 0 & 0 & 0 & 0 \\
\hline
\end{tabular}

\subsubsection{Phenolics and Flavonoids}

We recorded high total phenolic contents, in the range of 10.47 to $22.11 \mathrm{mg} / 100 \mathrm{~g} \mathrm{FW}$. In details, Ajwa Al Madinah had the highest content (22.11 mg/100 g DW), followed by Nabt Saif (22 mg/100 g DW), while Khla Al Qassim had the lowest content $(10.47 \mathrm{mg} / 100 \mathrm{~g}$ DW). Differences $(p<0.05)$ in total content of phenolics were observed among date varieties (Table 4). In this study, gallic, $p$-coumaric, and ferulic acid derivatives were the most dominant phenolic compounds, respectively. Moreover, different classes of flavonoids were identified in the tested varieties; quercetin, luteolin, apigenin, isoquercetrin, and rutin. Total flavonoid content was in the range of 1.22 and $2.82 \mathrm{mg} / 100 \mathrm{~g} \mathrm{DW}$, whereas Saffawy had the highest content (2.82 mg/100 g DW), followed by Ajwa Al Madinah (2.78 mg/100 g DW), and Al Qassim had the lowest content $1.22 \mathrm{mg} / 100 \mathrm{~g}$ DW (Table 5).

\subsubsection{Elemental Profiling (Macro- and Micronutrients)}

Our tested date cultivars contained significant amounts of minerals (Table 6). In particular, the potassium content was the highest (180.7-796.7 mg/100 g DW), followed in descending order by phosphorus (30.4-110.1 mg/100 g), magnesium (21.1-97.3 mg/100 g), and sodium (4.39-9.37 mg/100 g). Most of the analyzed minerals showed significant differences among the different cultivars; Khlas Al Kharj had the highest content of potassium (796.7 mg/100 g), magnesium (97.3 mg/100 g), sodium ( $9.3 \mathrm{mg} / 100 \mathrm{~g})$ and calcium $(0.919 \mathrm{mg} / 100 \mathrm{~g})$ as shown in Table 6, while Nabtit Ali and Sokary have the highest iron contents 1.648 and $1.644 \mathrm{mg} / 100 \mathrm{~g}$, respectively. 
Table 4. Concentrations of phenolic compounds (mg/100 g DW) in 12 Saudi date cultivars.

\begin{tabular}{|c|c|c|c|c|c|c|c|c|c|c|}
\hline Cultivars & Caffeic acid & Ferulic acid & Protocatechuic acid & Catechin & Gallic acid & $p$-Coumaric acid & Resorcinol & Chlorogenic acid & Syringic acid & Total phenolic \\
\hline Nabot Saif & $0.018 \pm 0.004$ & $1.94 \pm 0.42$ & $0.162 \pm 0.028$ & $0.574 \pm 0.12$ & $15.227 \pm 3.3$ & $3.275 \pm 0.720$ & $0.033 \pm 0.007$ & $0.2 \pm 0.044$ & $0.58 \pm 0.6$ & $22.00 \pm 5.35$ \\
\hline Rashodia & $0.013 \pm 0.001$ & $1.44 \pm 0.09$ & $0.115 \pm 0.007$ & $0.426 \pm 0.02$ & $11.312 \pm 0.7$ & $2.433 \pm 0.154$ & $0.025 \pm 0.002$ & $0.149 \pm 0.009$ & $0.66 \pm 0.0$ & $16.58 \pm 1.05$ \\
\hline Ajwa Al Madinah & $0.026 \pm 0.001$ & $2.52 \pm 0.11$ & $1.217 \pm 0.057$ & $0.526 \pm 0.02$ & $13.973 \pm 0.6$ & $3.087 \pm 0.004$ & $0.030 \pm 0.002$ & $0.184 \pm 0.009$ & $0.82 \pm 0.0$ & $22.11 \pm 1.10$ \\
\hline Khodry & $0.024 \pm 0.005$ & $2.56 \pm 0.57$ & $1.094 \pm 0.243$ & $0.473 \pm 0.10$ & $12.564 \pm 2.7$ & $2.702 \pm 0.601$ & $0.028 \pm 0.006$ & $0.165 \pm 0.037$ & $0.63 \pm 0.2$ & $20.13 \pm 4.21$ \\
\hline Khlas Al Ahsa & $0.018 \pm 0.004$ & $1.94 \pm 0.42$ & $0.527 \pm 0.588$ & $0.353 \pm 0.07$ & $9.370 \pm 2.05$ & $2.015 \pm 0.443$ & $0.021 \pm 0.005$ & $0.123 \pm 0.027$ & $0.55 \pm 0.1$ & $14.92 \pm 3.75$ \\
\hline Sokary & $0.019 \pm 0.003$ & $2.01 \pm 0.34$ & $0.893 \pm 0.119$ & $0.386 \pm 0.05$ & $10.24 \pm 1.36$ & $2.309 \pm 0.324$ & $0.022 \pm 0.004$ & $0.135 \pm 0.018$ & $0.60 \pm 0.0$ & $17.10 \pm 2.84$ \\
\hline Saffawy & $0.026 \pm 0.001$ & $2.52 \pm 0.11$ & $1.217 \pm 0.057$ & $0.526 \pm 0.02$ & $13.973 \pm 0.6$ & $3.005 \pm 0.142$ & $0.030 \pm 0.002$ & $0.184 \pm 0.009$ & $0.82 \pm 0.0$ & $21.99 \pm 1.27$ \\
\hline Khlas Al Kharj & $0.024 \pm 0.005$ & $2.56 \pm 0.57$ & $1.094 \pm 0.243$ & $0.333 \pm 0.09$ & $8.829 \pm 2.48$ & $1.302 \pm 0.290$ & $0.013 \pm 0.003$ & $0.080 \pm 0.018$ & $0.74 \pm 0.1$ & $14.97 \pm 1.28$ \\
\hline Mabroom & $0.018 \pm 0.004$ & $1.94 \pm 0.42$ & $0.527 \pm 0.588$ & $0.353 \pm 0.07$ & $9.370 \pm 2.05$ & $0.971 \pm 0.213$ & $0.010 \pm 0.002$ & $0.059 \pm 0.013$ & $0.55 \pm 0.1$ & $13.80 \pm 3.50$ \\
\hline Khla Al Qassim & $0.013 \pm 0.001$ & $1.44 \pm 0.09$ & $0.606 \pm 0.038$ & $0.262 \pm 0.01$ & 6.9610 .441 & $0.721 \pm 0.046$ & $0.008 \pm 0.001$ & $0.044 \pm 0.003$ & $0.41 \pm 0.0$ & $10.47 \pm 0.63$ \\
\hline Nabtit Ali & $0.019 \pm 0.003$ & $2.01 \pm 0.34$ & $0.893 \pm 0.119$ & $0.386 \pm 0.05$ & $10.246 \pm 1.3$ & $1.062 \pm 0.141$ & $0.011 \pm 0.001$ & $0.065 \pm 0.009$ & $0.60 \pm 0.0$ & $15.80 \pm 2.69$ \\
\hline Khals El Shiokh & $0.026 \pm 0.001$ & $2.52 \pm 0.11$ & $1.217 \pm 0.057$ & $0.526 \pm 0.02$ & $13.973 \pm 0.6$ & $1.448 \pm 0.068$ & $0.015 \pm 0.001$ & $0.089 \pm 0.004$ & $0.82 \pm 0.0$ & $20.37 \pm 1.17$ \\
\hline$p$ value & 0.00 & 0.00 & 0.00 & 0.00 & 0.00 & 0.00 & 0.00 & 0.00 & 0.049 & 0.00 \\
\hline
\end{tabular}

Table 5. Concentrations of flavonoid compounds (mg/100 g DW) in 12 Saudi date cultivars.

\begin{tabular}{ccccccc}
\hline Cultivars & Quercetin & Luteolin & Apigenin & Isoquercetrin & Rutin & Total Flavonoid \\
\hline Nabot Saif & $0.170 \pm 0.020$ & $0.045 \pm 0.010$ & $0.291 \pm 0.064$ & $0.726 \pm 0.160$ & $0.943 \pm 0.207$ & $2.175 \pm 0.461$ \\
Rashodia & $1.001 \pm 0.063$ & $0.033 \pm 0.002$ & $0.216 \pm 0.014$ & $0.540 \pm 0.034$ & $0.701 \pm 0.044$ & $2.491 \pm 0.158$ \\
Ajwa Al Madinah & $1.219 \pm 0.071$ & $0.041 \pm 0.002$ & $0.263 \pm 0.015$ & $0.411 \pm 0.001$ & $0.853 \pm 0.049$ & $2.787 \pm 0.138$ \\
Khodry & $1.112 \pm 0.247$ & $0.026 \pm 0.007$ & $0.240 \pm 0.053$ & $0.360 \pm 0.080$ & $0.547 \pm 0.154$ & $2.284 \pm 0.219$ \\
Khlas Al Ahsa & $0.536 \pm 0.597$ & $0.028 \pm 0.006$ & $0.179 \pm 0.039$ & $0.268 \pm 0.059$ & $0.580 \pm 0.128$ & $1.591 \pm 0.366$ \\
Sokary & $0.838 \pm 0.025$ & $0.028 \pm 0.001$ & $0.181 \pm 0.005$ & $0.271 \pm 0.008$ & $0.665 \pm 0.093$ & $1.983 \pm 0.104$ \\
Saffawy & $1.270 \pm 0.002$ & $0.041 \pm 0.002$ & $0.263 \pm 0.015$ & $0.394 \pm 0.023$ & $0.853 \pm 0.049$ & $2.821 \pm 0.088$ \\
Khlas Al Kharj & $1.112 \pm 0.247$ & $0.026 \pm 0.007$ & $0.081 \pm 0.023$ & $0.173 \pm 0.039$ & $0.547 \pm 0.154$ & $1.939 \pm 0.102$ \\
Mabroom & $0.536 \pm 0.597$ & $0.028 \pm 0.006$ & $0.086 \pm 0.019$ & $0.129 \pm 0.028$ & $0.580 \pm 0.128$ & $1.359 \pm 0.778$ \\
Khla Al Qassim & $0.616 \pm 0.039$ & $0.020 \pm 0.001$ & $0.064 \pm 0.004$ & $0.096 \pm 0.006$ & $0.431 \pm 0.027$ & $1.228 \pm 0.078$ \\
NabtitAIi & $0.950 \pm 0.133$ & $0.028 \pm 0.001$ & $0.087 \pm 0.003$ & $0.346 \pm 0.049$ & $0.665 \pm 0.093$ & $2.076 \pm 0.272$ \\
Khals El Shiokh & $1.219 \pm 0.071$ & $0.041 \pm 0.002$ & $0.127 \pm 0.007$ & $0.443 \pm 0.026$ & $0.853 \pm 0.049$ & $2.683 \pm 0.155$ \\
$p$ value & 0.00 & 0.00 & 0.00 & 0.00 & 0.00 & 0.00 \\
\hline
\end{tabular}


Table 6. Concentrations of minerals (mg/100 g DW) in 12 Saudi date cultivars.

\begin{tabular}{|c|c|c|c|c|c|c|c|c|c|c|}
\hline Cultivars & $\mathbf{K}$ & $\mathrm{Ca}$ & Mg & $\mathbf{P}$ & $\mathbf{N a}$ & $\mathbf{C u}$ & $\mathbf{F e}$ & Mn & Cd & Zn \\
\hline Nabot Saif & $431.88 \pm 27$ & $0.480 \pm 0.042$ & $50.814 \pm 3.09$ & $68.603 \pm 4.65$ & $5.48 \pm 0.609$ & $0.66 \pm 0.053$ & $0.27 \pm 0.022$ & $0.245 \pm 0.016$ & $0.002 \pm 0$ & $0.940 \pm 0.06$ \\
\hline Rashodia & $376.39 \pm 24$ & $0.410 \pm 0.036$ & $43.436 \pm 2.64$ & $55.960 \pm 3.79$ & $4.39 \pm 0.488$ & $2.62 \pm 0.212$ & $1.09 \pm 0.088$ & $0.196 \pm 0.013$ & $0.006 \pm 0$ & $0.75 \pm 0.05$ \\
\hline Ajwa Al Madinah & $290.025 \pm 4.6$ & $0.339 \pm 0.030$ & $35.941 \pm 2.18$ & $53.823 \pm 3.65$ & $7.01 \pm 0.782$ & $0.37 \pm 0.030$ & $0.15 \pm 0.013$ & $0.313 \pm 0.020$ & $0.001 \pm 0$ & $1.200 \pm 0.07$ \\
\hline Khodry & $463.502 \pm 6.9$ & $0.564 \pm 0.050$ & $59.738 \pm 3.63$ & $80.547 \pm 5.46$ & $6.52 \pm 0.725$ & $0.49 \pm 0.040$ & $0.20 \pm 0.017$ & $0.291 \pm 0.019$ & $0.001 \pm 0$ & $1.117 \pm 0.07$ \\
\hline Khlas Al Ahsa & $515.911 \pm 7.7$ & $0.637 \pm 0.056$ & $67.530 \pm 4.11$ & $110.170 \pm 7.4$ & $9.06 \pm 1.005$ & $0.57 \pm 0.046$ & $0.23 \pm 0.019$ & $0.404 \pm 0.026$ & $0.001 \pm 0$ & $1.550 \pm 0.10$ \\
\hline Sokary & $436.75 \pm 6.5$ & $0.512 \pm 0.045$ & $54.297 \pm 3.3$ & $80.640 \pm 5.46$ & $6.30 \pm 0.701$ & $3.94 \pm 0.319$ & $1.64 \pm 0.133$ & $0.281 \pm 0.018$ & $0.009 \pm 0$ & $1.077 \pm 0.07$ \\
\hline Saffawy & $387.4 \pm 5.8$ & $0.467 \pm 0.041$ & $49.442 \pm 3.01$ & $67.377 \pm 4.56$ & $5.40 \pm 0.601$ & $0.77 \pm 0.062$ & $0.32 \pm 0.026$ & $0.241 \pm 0.015$ & $0.002 \pm 0$ & $0.923 \pm 0.06$ \\
\hline Khlas Al kharj & $796.72 \pm 31.3$ & $0.919 \pm 0.081$ & $97.365 \pm 5.92$ & $63.887 \pm 4.33$ & $9.37 \pm 1.039$ & $0.70 \pm 0.057$ & $0.29 \pm 0.024$ & $0.418 \pm 0.027$ & $0.002 \pm 0$ & $1.603 \pm 0.10$ \\
\hline Mabroom & $396.95 \pm 15.6$ & $0.479 \pm 0.042$ & $50.808 \pm 3.09$ & $69.453 \pm 4.71$ & $5.85 \pm 0.65$ & $0.53 \pm 0.043$ & $0.22 \pm 0.018$ & $0.261 \pm 0.017$ & $0.001 \pm 0$ & $1.000 \pm 0.06$ \\
\hline Khla Al Qassim & $665.36 \pm 26.1$ & $0.783 \pm 0.069$ & $82.930 \pm 5.04$ & $57.083 \pm 3.87$ & $8.9 \pm 0.999$ & $0.65 \pm 0.053$ & $0.27 \pm 0.022$ & $0.401 \pm 0.026$ & $0.002 \pm 0$ & $1.537 \pm 0.1$ \\
\hline Nabtit Ali & $180.755 \pm 7.1$ & $0.200 \pm 0.018$ & $21.141 \pm 1.28$ & $30.470 \pm 2.06$ & $6.58 \pm 0.728$ & $3.95 \pm 0.319$ & $1.64 \pm 0.133$ & $0293 \pm 0.019$ & $0009 \pm 0$ & $1.127 \pm 0.07$ \\
\hline Khals El Shiokh & $486.383 \pm 19.1$ & $0.581 \pm 0.051$ & $61.581 \pm 3.74$ & $103.13 \pm 6.99$ & $8.6 \pm 0.953$ & $0.29 \pm 0.024$ & $0.12 \pm 0.010$ & $0.383 \pm 0.024$ & $0.001 \pm 0$ & $1.470 \pm 0.09$ \\
\hline$p$ value & 0.00 & 0.00 & 0.00 & 0.00 & 0.00 & 0.00 & 0.00 & 0.00 & 0.00 & 0.00 \\
\hline
\end{tabular}




\subsection{Principal Component Analysis (PCA) and Hierarchical Clustering (HCA)}

All the measured metabolites (amino acids, sugars, organic acids, phenolics, flavonoids, antioxidants, and macrominerals and trace elements) were subjected to principal component analysis (PCA) to identify differences in metabolite profiles among the studied date cultivars (Figure 2).

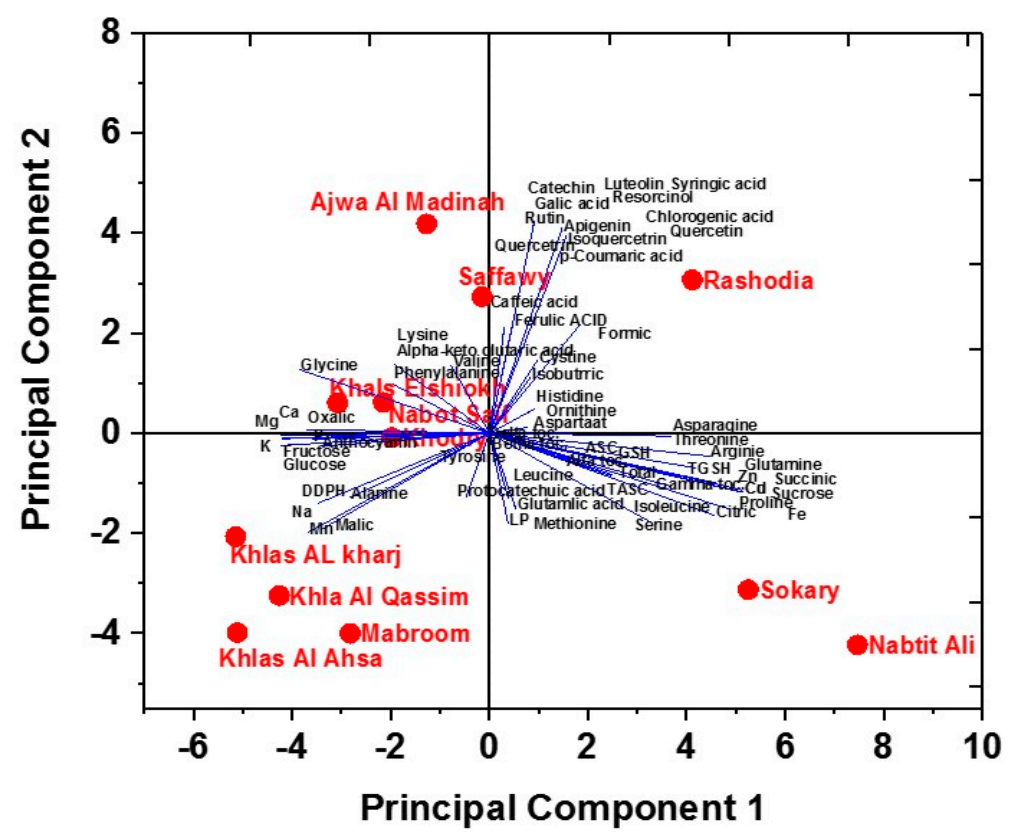

Figure 2. Principal component analysis (PCA) of metabolites in 12 Saudi date cultivars. Bi-plot of principle component $1(42.7 \%)$ and principle component $2(23.5 \%)$.

PCA revealed that the first two principal components (PC1 and PC2) accounted for $49.6 \%$ of the total variance within the data set. The first principal component PC1 explained $28.3 \%$, and the second principal component PC2 explained $21.3 \%$ of the data variation. These two principal components separated the studied cultivars into four different groups. The first group consisted of Sokary and Nabtit Ali cultivars, the second group of Khlas Al Kharj, Khla Al Qassim, Mabroom, Khlas Al Ahsa, the third group of Khals El Shiokh, Nabot Saif, Khodry, and the fourth group consisted of the Ajwa Al Madinah, Saffawy, Rashodia, Khals E1 Shiokh, Nabot Saif, and Khodry cultivars. PC1 showed loadings for Sokary and Nabtit Ali, whereas PC2 showed loadings for Ajwa Al Madinah, Saffawy, Khals El Shiokh and Nabot Saif Hierarchical cluster analysis (HCA) of measured metabolites was also performed. A heat map of the metabolite profiling (Figure 3). At a first glance at the entire data set, it is clear that some of the changes in metabolites (aspartate and beta-tocopherol) are almost similar in all cultivars. HCA indicated two main clusters; the first cluster Sokary, Rashodia and Nabtit Ali and the second cluster contained other tested cultivars. This separation could be explained by the lower content of GSH, succinic and citric acids, some amino acids (isoleucine, serine, arginine, proline and glutamine), and some minerals $(\mathrm{Cd}$, $\mathrm{Zn}, \mathrm{Fe}$ and $\mathrm{Cu}$ ) in date cultivars of second cluster. Khlas Al Ahsa, Khlas Al kharj, Khlas Al Qassim and Khlas Al Shiokh showed high levels of mono-saccharides (glucose and fructose) and minerals (K, Na, Mg, $\mathrm{Mn}$ and $\mathrm{Ca}$ ), whereas Khlas Al Kharj showed the highest levels. Oppositely, lower levels of resorcinol, chlorogenic acid, coumaric acid, isoquercetin and apignin were recorded in in all Khlas varieties (Al Asha, Al Kharj, El Shiokh). 


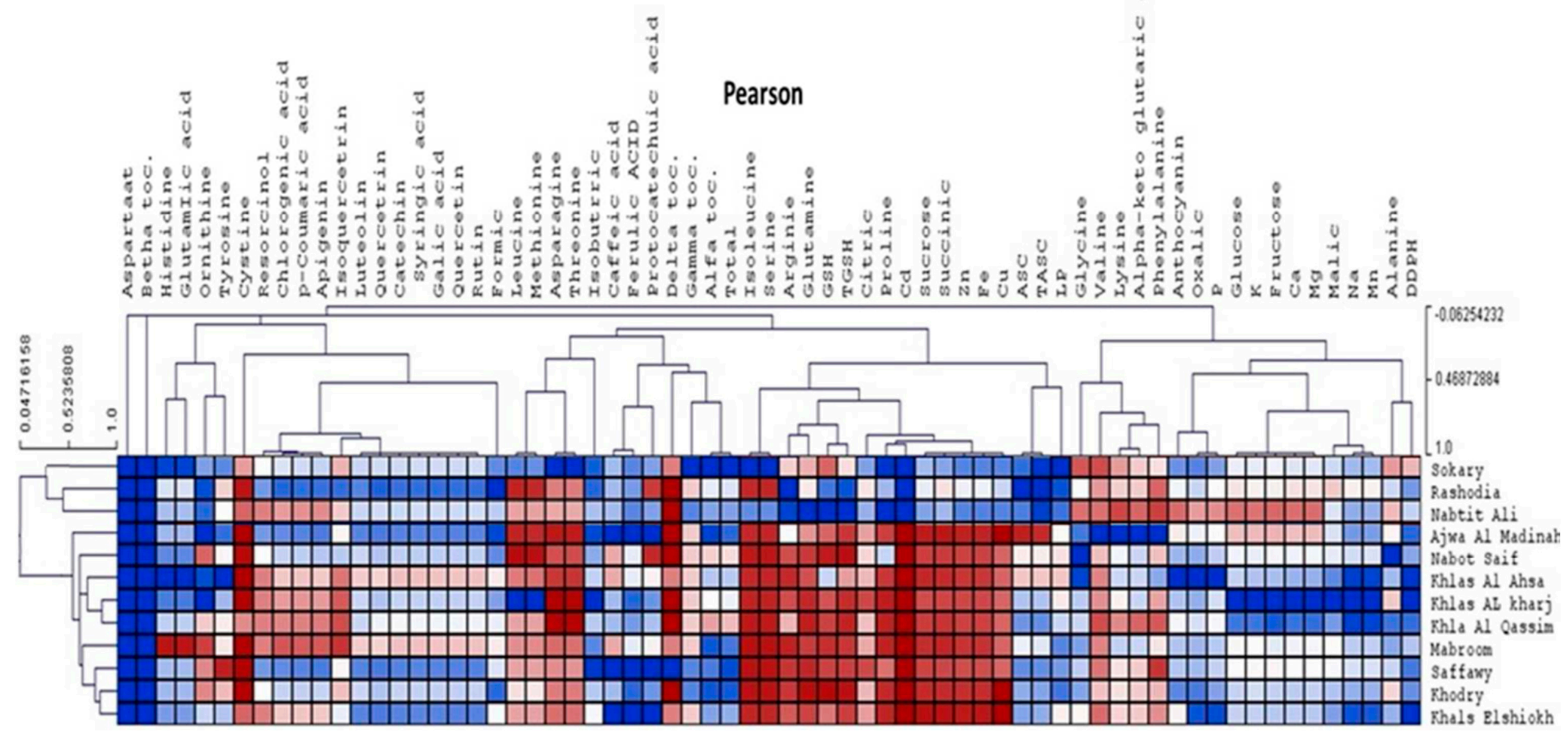

Figure 3. Heat maps of the metabolite profiles of 12 Saudi date cultivars. A total of 42 metabolites were quantified by high performance liquid chromatography for each cultivar, including amino acids, organic acids, sugars, phenolic compounds, glutathione and vitamins. 


\subsection{Biological Activity}

\subsubsection{DPPH (1,1-Diphenyl-2-picrylhydrazyl) Free Radical Scavenging Activity}

DPPH scavenging activity of fruit extracts of different palm date varieties was investigated. Overall, all date varieties showed DPPH scavenging ability (Figure 1a). In particular, Khlas El shiokh, Khlas Al Ahsa, Khlas Al Kharj showed the strongest DPPH scavenging capacity ( $>38 \%)$, whereas Sokary cultivar had the lowest value (14\%).

\subsubsection{Anti-lipid Peroxidation Assay}

Our results showed that almost all the tested palm date extracts inhibited lipid peroxidation (Figure 1b), however extract of Rashodia, Khodry, Sokary, Saffawy, Mabroom and Nabtit Ali showed a higher scavenging potential (i.e., $\mathrm{IC}_{50}>2.0$ ). Although Sokary variety showed the highest lipid peroxidation inhibition activity, its DPPH was very low. The higher activity of Sokary extract could be explained by its higher vitamins (ascorbate, tocopherols, Figure 4), total phenolics and flavonoids (Tables 4 and 5).

\section{(a)}

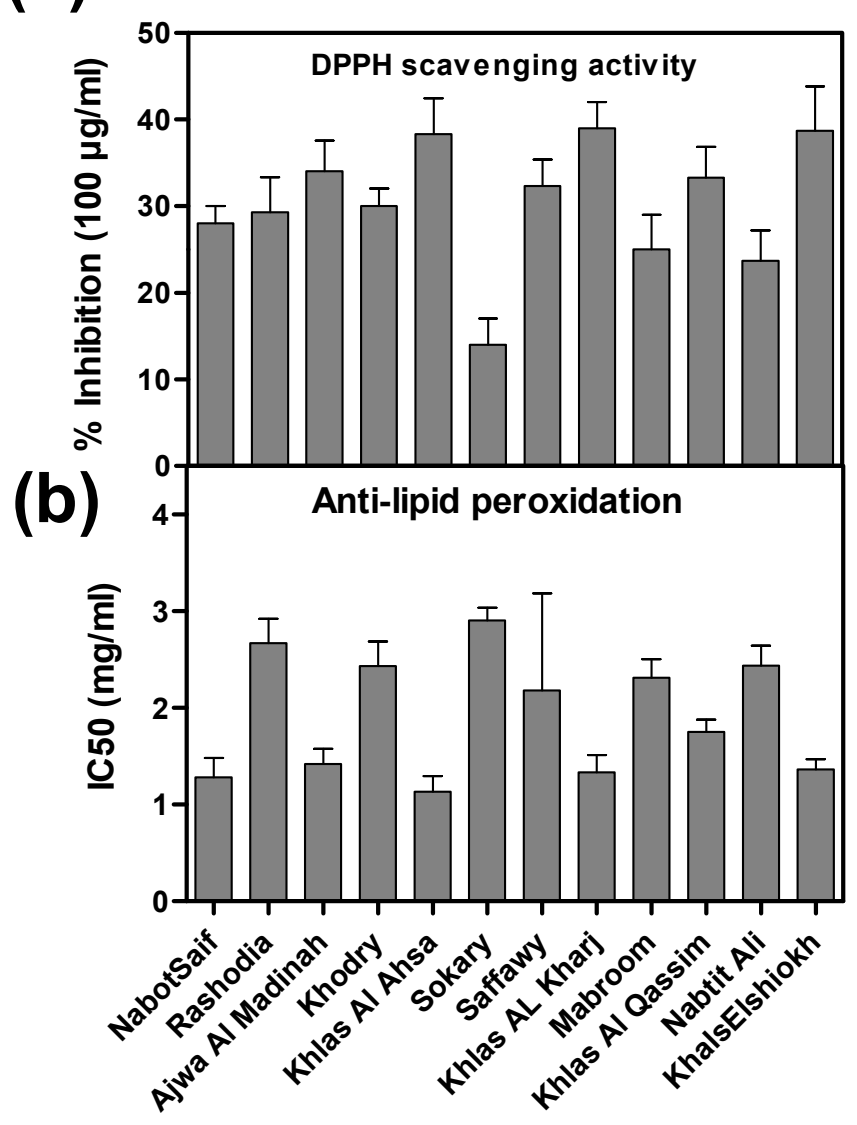

Figure 4. Antioxidant activity: DPPH scavenging activity and anti-lipid peroxidation activity of 12 Saudi date cultivars. (a) DPPH (1,1-diphenyl-2-picrylhydrazyl) free radical scavenging activity; (b) anti-lipid peroxidation. 


\subsection{Discussion}

In this study, the chemical composition and biological activity of 12 date varieties which represent different geographical locations of Saudi Arabia were evaluated. All the measured metabolites (amino acids, sugars, organic acids, phenolics, flavonoids, antioxidants, macrominerals and trace elements) were subjected to principal component analysis (PCA) and hierarchical cluster analysis (HCA) to identify differences in metabolite profiles among cultivars; PCA is widely used to assess the differences between plant varieties/cultivars at the metabolic level. The similarities and variation observed among studied date cultivars in their chemical composition can be explained on the basis of their different metabolic responses and environmental conditions. Indeed metabolic profiles of many crops are significantly affected by genotypes and growing location [30-32].

The 2,2-diphenyl-1-picrylhydrazyl (DPPH) radical-scavenging ability assay is widely used to evaluate the free radical scavenging capacity of antioxidants [33]. The decrease in the level of free radicals with the increase in the concentration of the palm date extracts indicates their role extract as antioxidants. High inhibition of DPPH radical formation was also recorded for the Deglet Noor cultivar (about 54\%) [34]. The DPPH scavenging ability of date palm extract could be explained by higher availability of different antioxidants. Similar to our results, $[7,35]$ indicated that palm dates contained flavonoids, such as luteolin, quercetin, and apigenin, as well as phenolics, such as $p$-coumaric, ferulic, and sinapic acids, and cinnamic acid derivatives. Accumulation of free radicals can damage cells at the level of nucleic acids, membrane lipids, and proteins, leading to generation of cancer and aging related diseases [36,37]. A strong correlation between the antioxidant activity and the total phenolic and total flavonoids of palm dates was also recorded [38].

The nutritional quality of date palm could, in part, be associated with their major constituents such as flavonoid compounds, phenolics, sugars, amino and organic acids [39-44]. Similarly, in our study, higher antimicrobial activity of date palm may be due the presence of phenolics, flavonoids and terpenoids.

Amino acid profiles revealed that all tested date cultivars contained the majority of essential amino acids: lysine, isoleucine, leucine, methionine, threonine, valine, histidine and phenylalanine. This observation was in accordance with those reported by Bouaziz et al. [45], where amino acid composition of Tunisian date seeds was profiled; however, lysine presented the largest amount among these Saudi date cultivars. The disappearance of tryptophan could be attributed to its destruction during acid hydrolysis that could also account for the damage to cysteine [46].

Dates contain a high concentration of sugars, which are considered the main component. These carbohydrates are mainly reducing sugars in the form of glucose, fructose, mannose and maltose and non-reducing sugars (primarily sucrose), as well as small amounts of polysaccharides (such as cellulose and starch) [47]. The difference in sugar composition reflects the difference in invertase activity in these cultivars, which causes reduction in sucrose content [3]. Our results are lower than those observed by Al-Farsi et al. [16], who studied the compositional and sensory characteristics of three native sun-dried date varieties grown in Oman and reported that total sugar content ranged from 56.1 to $62.2 \mathrm{~g} / 100 \mathrm{~g}$; this is can be explained by non-enzymatic browning during storage (Maillard reaction) [48].

Besides nutritional value, the presence of taste-active components such as organic acids can improve the sensory characteristics of products as they are responsible for the sour, tart, acidic, and characteristic fruity tastes of many foods. Organic acids also influence the growth of microorganisms in fruit and 
therefore affect the storage quality of the product. Another aspect of organic acids is their influence on the sensory properties of dates. Similarly, the study of Al-Farsi et al. [16] indicated that malic acid is the predominant organic acid in dates and has a characteristic fruity, mellow, smooth, tart, and sour taste in fresh fruits. Moreover, the presence and composition of organic acids may be affected by various factors such as variety, growing conditions, maturity, season, geographic origin, fertilization, soil type, storage conditions, amount of sunlight received, and time of harvest, among others [49].

Dates have high content of polyphenolic compounds, which explains their antioxidant activity $[7,8]$. The amount of total phenolics detected in the tested cultivars came more or less close to that detected by Messaoudi et al. [50], who reported that the total phenolic content ranged between 27.2 and $38.5 \mathrm{mg} / 100 \mathrm{~g} \mathrm{FW}$ for the methanolic date extracts and between 22.8 and $42.6 \mathrm{mg} / 100 \mathrm{~g}$ FW for the corresponding ethyl acetate extracts, however, Al-Farsi et al. [51] reported total phenolics content values between 217.0 to $343.0 \mathrm{mg} / 100 \mathrm{~g}$ fresh weight for some Omani dates. We found nine phenolics i.e., caffeic, ferulic, protocatechuic, catechin, gallic, $p$-coumaric, resorcinol, chlorogenic and syringic acids. Similarly, Al-Farsi et al. [51] found nine phenolics in the Omanian date varieties, with $p$-hydroxy-benzoic, protocatechuic, and $m$-coumaric acids as the main components. The heterogeneity of these results could be attributed to several factors - the origin of the plant, the variety, the extraction procedure, and the measurement method [50]. Previous studies also showed that date palm contained flavonoids, such as luteolin, quercetin, and apigenin [38].

Ascorbic acid (ASC), glutathione (GSH) and tocopherol contents in the fruits of the 12 date cultivars were evaluated. Tocopherol also varied significantly among the date cultivars. There are various forms of tocopherols $(\alpha, \beta, \gamma, \delta)$ and we observed that $\alpha$-tocopherol was the main contributor for the change in total tocopherol content, as also observed by $[52,53]$. To our knowledge there are no previous reports measuring ascorbate, glutathione and tocopherol contents in these dates. As we observed significant variation in the contents of antioxidant molecules among the date cultivars, these results have positive implications for breeding programs aimed at increasing the levels of antioxidant compounds in commercial genotypes. These antioxidant molecules play an important role in defense against oxidative stress [54], and also have nutritional value [55].

The results indicated that tested dates cultivars contained significant amounts of minerals, and previous studies [55-57] also reported higher contents of minerals, which are three to five times the amounts found in grapes, apples, oranges and bananas [58]. These results confirm that dates have high nutritional value. The high potassium and low sodium contents of dates are suitable for people with hypertension [59]. Similarly, $[60,61]$ reported that dates are a very good source of many minerals which are important for metabolism in human cells. For instance, magnesium and calcium are essential for healthy bone development and for energy metabolism, and iron is essential for red blood cell production.

\section{Experimental Section}

\subsection{Sample Collection and Extract Preparation}

Fresh dates were collected from cultivars grown in the Kingdom of Saudi Arabia. Fruits were collected at the tamr stage from retail local markets in Sakaka city, Aljouf. The local Arabic names of the cultivars used in this study are: Nabot Saif, Rashodia, Ajwa Al Madinah, Khodry, Khlas Al Ahsa, 
Sokary, Saffawy, Khlas Al Kharj, Mabroom, Khlas Al Qassim, Nabtit Ali and Khals El Shiokh. The dates were washed and stored at $-20{ }^{\circ} \mathrm{C}$ for further analysis.

\subsection{Biological Activity}

\subsubsection{DPPH Free Radical Scavenging Assay}

The 1,1-diphenyl-2-picrylhydrazyl (DPPH) free radical scavenging activity of date fruit extracts was estimated according to the method explained by Cheung et al. [62] with some modifications. Briefly, the crude extracts were resuspended in methanol, and aliquots of $0.2 \mathrm{mM} \mathrm{DPPH}$ in methanol $(160 \mu \mathrm{L})$ were mixed with each extract $\left(40 \mu \mathrm{L}, 0.01-1 \mathrm{mg} \cdot \mathrm{mL}^{-1}\right)$. The mixtures were left under subdued light for $10 \mathrm{~min}$. The absorbance at $520 \mathrm{~nm}$ was measured against a blank. The radical scavenging activity was measured as a decrease in the absorbance of DPPH and was calculated using the following equation:

$$
\text { Scavenged DPPH }=\left[1-\left(\text { Asample }_{-} \text {Asample blank }\right) / A_{\text {control }} \times 100\right]
$$

where Acontrol, $\mathrm{A}_{\text {sample, and }}$ Asample blank represent the absorbance of the control group (160 $\mu \mathrm{L} 0.2 \mathrm{mM} \mathrm{DPPH}$ and $40 \mu \mathrm{L}$ methanol or water), sample group (160 $\mu \mathrm{L} 0.2 \mathrm{mM} \mathrm{DPPH}$ and $40 \mu \mathrm{L}$ extract or reference compounds (Trolox)), and sample blank (160 $\mu \mathrm{L}$ methanol and $40 \mu \mathrm{L}$ extract or reference compounds), respectively.

\subsubsection{Anti-Lipid Peroxidation Assay}

Lipid peroxidation was determined as described by Patro et al. [63] with some modifications. Briefly, liposomes were prepared by sonicating a mixture of phosphatidyl choline (300 mg) in $10 \mathrm{mM}$ phosphate buffer (30 mL, $\mathrm{pH} 7.4$ ) on ice for $2 \mathrm{~h}$. To an aliquot of potassium phosphate buffer (total volume $1 \mathrm{~mL}$ ) at $\mathrm{pH} 7.4(10 \mathrm{mM})$, the liposomes $(250 \mu \mathrm{L})$, and extract (resuspended in methanol) or methanol $(450 \mu \mathrm{L})$, was added $\mathrm{FeCl}_{2}, \mathrm{H}_{2} \mathrm{O}_{2}$ and ascorbic acid, each to a final concentration of $125 \mu \mathrm{M}$. After incubating the mixture at $30{ }^{\circ} \mathrm{C}$ for $4 \mathrm{~h}, 250 \mu \mathrm{L}$ of the final mixture was added to TCA-TBA-HCl reagent $(500 \mu \mathrm{L}$, $15 \% w / v, \mathrm{TCA} ; 0.375 \% w / v, \mathrm{TBA} ; 0.25 \mathrm{M} \mathrm{HCl})$. After that the mixture was heated at $100{ }^{\circ} \mathrm{C}$ on a boiling water bath for $15 \mathrm{~min}$, followed by centrifugation at $3000 \mathrm{~g}$ for $5 \mathrm{~min}$. The absorbance of the supernatant was measured at $532 \mathrm{~nm}$ against a blank.

\subsection{Metabolic Profiling}

\subsubsection{Amino Acids}

Fresh dates were homogenized by using a MagNALyser (Roche, Vilvoorde, Belgium) for 1 min, at $7000 \mathrm{rpm}$, in $80 \%(v / v)$ aqueous ethanol $(1 \mathrm{~mL})$. Samples were spiked with norvaline as a control for the loss of amino acids during extraction. The homogenate was centrifuged at 14,000 rpm for $20 \mathrm{~min}$, the supernatant was evaporated under vacuum, and the pellet was resuspended in chloroform $(1 \mathrm{~mL})$. Simultaneously, the residue was reextracted with HPLC grade water $(1 \mathrm{~mL})$ using a MagNALyser (Roche, Vilvoorde, Belgium) and the supernatant after centrifugation (14,000 rpm for $20 \mathrm{~min}$ ) was mixed with the pellet suspended in chloroform. Then they were centrifuged for $10 \mathrm{~min}$ at 14,000 rpm and the aqueous phase was filtered using Millipore micro filters $(0.2 \mu \mathrm{M}$ pore size $)$ before assaying free 
amino acids (FAA) levels. Amino acids were determined by using a Waters Acquity UPLC-tqd system (Milford, Worcester County MA, USA) equipped with a Sinhaa BEH amide $2.1 \times 50$ column [64].

\subsubsection{Sugars}

Sugar levels were measured according to Alasalvar et al. [65] using high-performance liquid chromatography (HPLC). Sugars were extracted from dates with acetonitrile/water $(2 \mathrm{~mL}, 1: 1, v / v)$ for $2 \mathrm{~min}$. The extract was then kept in a water bath at $55-60^{\circ} \mathrm{C}$ for $15 \mathrm{~min}$ (stirring frequently with a glass rod to aid in dissolving the sugars) and subsequently filtered through a Whatman No. 541 filter paper. After that, another $20 \mathrm{~mL}$ of solvent was added to the remaining pulp, and the extraction was repeated three times. Finally, all combined supernatants were collected and made up to a final volume of $100 \mathrm{~mL}$ with the extraction solvent. Column temperature and injection volume were set at $30^{\circ} \mathrm{C}$ and $20 \mu \mathrm{L}$, respectively. The mobile phase (filtered through a $0.45 \mu \mathrm{m}$ Millipore filter and degassed prior to use) was a mixture of acetonitrile and HPLC-grade water at a ratio of 75:25 $(v / v)$ at $1 \mathrm{~mL} \cdot \mathrm{min}^{-1}$. Identified sugars were quantified on the basis of peak areas and comparison with a calibration curve obtained with the corresponding standards ranging from 1 to $10 \mathrm{mg} / 100 \mathrm{~mL}$ of acetonitrile/water $(1: 1, v / v)$. Sugars were expressed as milligrams per $100 \mathrm{~g}$ of fresh weight $(\mathrm{mg} / 100 \mathrm{~g} \mathrm{FW})$.

\subsubsection{Organic Acids}

Palm fruit samples (500 mg FW) were homogenized in phosphoric acid $(0.1 \%$; containing $0.003 \%$ butylated hydroxyanisole) by using a MagNALyser. The extract was centrifuged at 14,000 rpm for $30 \mathrm{~min}$ at $4{ }^{\circ} \mathrm{C}$. The supernatants were passed through Millipore micro filters $(0.2 \mu \mathrm{M}$ pore size $)$. Organic acids were detected by HPLC using a SUPELCOGEL C-610H column $(300 \mathrm{~mm} \times 7.8 \mathrm{~mm}$, Supelco, Sigma, St. Louis, MO, USA) coupled to UV detection system set at $210 \mathrm{~nm}$ (LaChrom L-7455 diode array, LaChrom, Tokyo, Japan). The mobile phase was a $0.1 \%$ phosphoric acid at a flow rate of $0.45 \mathrm{~mL} \cdot \mathrm{min}^{-1}$. Organic acids were quantified using a calibration curve obtained with the corresponding standards.

\subsubsection{Phenolics}

Date fruit samples (50 g DW) were manually separated from the seed, crushed and cut to small pieces with a sharp knife and dry-blended for 3 min with a blender. The date fruit was then extracted with acetone-water $(250 \mathrm{~mL}, 4: 1, v / v)$, at room temperature for $24 \mathrm{~h}$ using an orbital shaker. The extracts were then filtered and centrifuged (Hettich Zentrifugen, Tuttlingen, Germany) at $4000 \mathrm{~g}$, for $10 \mathrm{~min}$ and the supernatant was concentrated under reduced pressure at $40{ }^{\circ} \mathrm{C}$ for $3 \mathrm{~h}$ using a rotary evaporator (IKA-WERKE-RV06ML, Stanfer, Germany) to obtain the DPF hydoxyacetone crude extract. The residues were dissolved in $\mathrm{HPLC}$ grade $\mathrm{MeOH}$ to give $1000 \mathrm{mg} \cdot \mathrm{L}^{-1}$ and measured as mentioned previously by Gomaa and AbdElgawad [66]. Briefly methanol-dissolved sample (20 $\mu \mathrm{L})$ was injected into a Shimadzu HPLC system (SCL-10 A vp, Shimadzu Corporation, Kyoto, Japan). The HPLC system consisted of a diode-array detector and a Lichrosorb Si-60, $7 \mu \mathrm{m}, 3 \times 150 \mathrm{~mm}$ column. The mobile phase consisted of water/formic acid, 90:10, $v / v$; and acetonitrile/water/formic acid, 85:10:5, $v / v / v$. Tentatively identified phenolic acids and flavonoids were quantified with a calibration curve obtained with the corresponding standards. The results were expressed as mg/100 g DW. 


\subsubsection{Ascorbate, Glutathione and Tocopherols}

Glutathione and ascorbate content was determined by reversed phase HPLC separation, followed by UV detection according to the method described by Potters et al. [67]. Total antioxidant concentration (reduced + oxidized) was determined after reduction with 0.04 M DTT for $10 \mathrm{~min}$ at room temperature, and the redox status was calculated as the ratio of the reduced form to the total concentration. Tocopherols were extracted with hexane, and measured according to the method of Siebert 1999 [68]. The extract was vaccum dried (CentriVap concentrator, Labconco, KS, USA) and was re-suspended in hexane. Tocopherols were separated and quantified by HPLC (Shimadzu, Hertogenbosch, Netherlands) using normal phase conditions (Particil Pac $5 \mu \mathrm{m}$ column material, length $250 \mathrm{~mm}$, i.d. $4.6 \mathrm{~mm}$ ). 5,7-dimethyltocol (DMT; 5 ppm) was used as an internal standard. Data were analyzed with Shimadzu Class VP 6.14 software provided by the HPLC system (Shimadzu, Tokyo, Japan).

\subsubsection{Macro-Minerals and Trace Elements}

Date fruits were digested in a 5:1 ratio of $\mathrm{HNO}_{3} / \mathrm{H}_{2} \mathrm{O}$ in an oven and macro-minerals and trace elements were determined by mass spectrometry (ICP-MS, Finnigan Element XR, Scientific, Bremen, Germany) according to Agusa et al. [69]. A mixture of standards was prepared in 1\% nitric acid.

\subsection{Statistical Analysis}

The data were analyzed by procedure of the Statistical Analysis System (SPSS Inc., Chicago, IL, USA). The assumptions of normality of distribution and homogeneity of variance were examined. Since both assumptions were met, transformations were not necessary and analysis of variance (ANOVA) was done on the original data. The differences between cultivars were tested by one-way ANOVA procedure. Number of replicates for each cultivar were three $(n=3)$. The significant differences between the means were determined by using the Duncan test $(p<0.05)$. Principal Component Analysis (PCA) was performed by using OriginLab software (9, OriginLab, Northampton, MA, USA). Cluster analysis was performed by using Pearson distance metric by using MultiExperiment Viewer $(\mathrm{MeV})^{\mathrm{TM}} 4$ software package (version 4.5, Dana-Farber Cancer Institute, Boston, MA, USA). All parameters and cultivars were included in the analysis.

\section{Conclusions}

The differences in the chemical composition of date fruits altered their nutritional value and biological activities. Our study showed that the date fruit extracts from different cultivars have different free radical scavenging and anti-lipid peroxidation activities. The similarities and chemical composition variations observed among the studied date cultivars could explained the variation in date fruit biological activity. Future studies exploring the link between dates' nutritional quality and their growth climate conditions are needed. 


\section{Acknowledgments}

This work was funded by Y.A. Jameel Scientific Chair of Prophetic Medical Applications, King Abdulaziz University, Kingdom of Saudi Arabia, under grant no. (PM 0002 (2.1)-1434). The authors, therefore, acknowledge with thanks Y.A. Jameel Scientific Chair of Prophetic Medical Applications technical and financial support.

\section{Author Contributions}

Ismail Hamad, Hamada AbdElgawad, Han Asard, Soad Al Jaouni, Nashwa Hagagy and Samy Selim designed the research; Hamada AbdElgawad, Momtaz Hegab and Samy Selim performed the experimental work; Hamada AbdElgawad, Gaurav Zinta, Sherif Hassan and Samy Selim wrote the manuscript. All authors discussed, edited and approved the final version.

\section{Conflicts of Interest}

The authors declare no conflict of interest.

\section{References}

1. Chao, C.T.; Krueger, R.R. The date palm (Phoenix dactylifera L.): Overview of biology, uses, and cultivation. Hort. Sci. 2007, 42, 1046-1311.

2. Awad, M.A. Increasing the rate of ripening of date palm fruit (Phoenix dactylifera L.) cv. Helali by preharvest and postharvest treatments. Postharvest Biol. Technol. 2007, 43, 121-127.

3. Fayadh, J.M.; Al-showiman S.S. Chemical composition of date palm (Phoenix dactylifera L.). J. Chem. Soc. Pakistan 1990, 12, 84-103.

4. Besbes, S.; Blecker, C.; Deroanne, C.; Drira, N.E.; Attia, H. Date seeds: Chemical composition and characteristic profiles of the lipid fraction. Food Chem. 2004, 84, 577-584.

5. Al-Shahib, W.; Marshall, R.J. The fruit of the date palm: Its possible use as the best food for the future? Int. J. Food Sci. Nutr. 2003, 54, 247-259.

6. Vayalil, P.K. Antioxidant and antimutagenic properties of aqueous extract of date fruit (Phoenix dactylifera L. Arecaceae). J. Agric. Food Chem. 2002, 50, 610-617.

7. Mansouri, A.; Embarek, G.; Kokkalou, E.; Kefalas, P. Phenolic profile and antioxidant activity of the Algerian ripe date palm fruit (Phoenix dactylifera). Food Chem. 2005, 89, 411-420.

8. Al-Turki, S.; Shahba, M.A.; Stushnoff, C. Diversity of antioxidant properties and phenolic content of date palm (Phoenix dactylifera L.) fruits as affected by cultivar and location. J. Food Agric. Environ. 2010, 8, 253-260.

9. Al-Farsi, M.; Alasalvar, C.; Al-Abid, M.; Al-Shoaily, K.; Al-Amry, M.; Al-Rawahy, F. Compositional and functional characteristics of dates, syrups, and their by-products. Food Chem. 2007, 104, 943-947.

10. Yousif, A.K.; Benjamin, N.D.; Kado, A.; Alddin, S.M.; Ali, S.M. Chemical composition of four Iraqi date cultivars. Date Palm J. 1982, 1, 285-294.

11. Burt, S. Essential oils: Their antibacterial properties and potential applications in foods - A review. Int. J. Food Microbiol. 2004, 94, 223-253. 
12. Sallal, A.K.; Ashkenani, A. Effect of date extract on growth and spore germination of Bacillus subtilis. Microbios 1989, 59, 203-210.

13. Shraideh, Z.A.; Abu-Elteen, K.H.; Sallal, A.K.J. Ultrastructural effects of date extract on Candida albicans. Mycopathologia 1998, 142, 119-123.

14. Selim, S.A.; Alfy, S.E.; Al-Ruwaili, M.; Abdo, A; Jaouni, S.A. Susceptibility of imipenem-resistant Pseudomonas aeruginosa to flavonoid glycosides of date palm (Phoenix dactylifera L.) tamar Growing in Al Madinah, Saudi Arabia. Afr. J. Biotechnol. 2012, 11, 416-422.

15. Abdul, J.C.; Shyam, S.K.; Sreeramanan, S. Variations in hormones and antioxidant status in relation to flowering in early, mid, and late varieties of date palm (Phoenix dactylifera) of United Arab Emirates. Sci. World J. 2015, 2015, doi:10.1155/2015/846104.

16. AbdElgawad, H.; Farfan-Vignolo, E.R.; de Vos, D.; Asard, H. Elevated $\mathrm{CO}_{2}$ mitigates drought and temperature-induced oxidative stress differently in grasses and legumes. Plant Sci. 2015, 231, 1-10.

17. AbdElgawad, H.; de Vos, D.; Zinta, G.; Domagalska, M.A.; Beemster, G.T.S; Asard H. Grassland species differentially regulate proline concentrations under future climate conditions: An integrated biochemical and modelling approach. New Phytol. 2015, doi:10.1111/nph.13481.

18. AbdElgawad, H.; Peshev, D.; Zinta, G; van den Ende, W.; Janssens, I.A.; Asard, H. Climate extreme effects on the chemical composition of temperate grassland species under ambient and elevated $\mathrm{CO}_{2}$ : A comparison of fructan and non-fructan accumulators. PLoS ONE 2014, 9, e92044.

19. Pandey, R.; Zinta G.; AbdElgawad, H.; Ahmad, A.; Jain, V.; Janssens, I.A. Physiological and molecular alterations in plants exposed to high $\mathrm{CO}_{2}$ under phosphorus stress. Biotechnol. Adv. 2015, 33, 303-316.

20. Zinta, G.; AbdElgawad, H.; Domagalska, M.A.; Vergauwen, L.; Knapen, D.; Nijs, I.; Janssens, I.A.; Beemster, G.T.; Asard, H. Physiological, biochemical, and genome-wide transcriptional analysis reveals that elevated $\mathrm{CO}_{2}$ mitigates the impact of combined heat wave and drought stress in Arabidopsis thaliana at multiple organizational levels. Glob. Change Biol. 2014, 20, 3670-3685.

21. El-Shafey, N.M.; Abd-Elgawad, H. Luteolin, a bioactive flavone compound extracted from Cichorium endivia L. subsp. divaricatum alleviates the harmful effect of salinity on maize. Acta Physiol. Plant. 2012, 34, 2165-2177.

22. El-Soud, W.A.; Hegab, M.M.; AbdElgawad, H.; Zinta, G.; Asard, H. Ability of ellagic acid to alleviate osmotic stress on chickpea seedlings. Plant Physiol. Biochem. 2013, 71, 173-183.

23. Farag, M.A.; Mohsen, M.; Heinke, R.; Wessjohann, L.A. Metabolomic fingerprints of 21 date palm fruit varieties from Egypt using UPLC/PDA/ESI-qTOF-MS and GC-MS analyzed by chemometrics. Food Res. Int. 2014, 64, 218-226.

24. Al-Farsi, M.; Alasalvar, C.; Morris, A.; Baron, M.; Shahidi, F. Compositional and sensory characteristics of three native sun-dried date (Phoenix dactylifera L.) varieties grown in oman. J. Agric. Food Chem. 2005, 53, 7586-7591.

25. Singh, V.; Guizani, N.; Essa, M.M.; Hakkim, F.L.; Rahman, M.S. Comparative analysis of total phenolics, flavonoid content and antioxidant profile of different date varieties (Phoenix dactylifera $\mathrm{L}$.) from Sultanate of Oman. Int. Food Res. J. 2012, 19, 1063-1070.

26. Allaith, A.A.A. Antioxidant activity of Bahraini date palm (Phoenix dactylifera L.) fruit of various cultivars. Int. J. Food Sci. Technol. 2008, 43, 1033-1040. 
27. Okada, T.; Nakamura, Y.; Kanaya, S.; Takano, A.; Malla, K.J.; Nakane, T.; Kitayama, M; Sekita, S. Metabolome analysis of ephedra plants with different contents of ephedrine alkaloids by using UPLC-Q-TOF-MS. Planta Med. 2009, 75, 1356-1362.

28. Saafi, E.B.; Louedi, M.; Elfeki, A.; Zakhama, A.; Najjar, M.F.; Hammami, M.; Achour, L. Protective effect of date palm fruit extract (Pheonix dactylifera $\mathrm{L}$.) on dimethoate induced-oxidative stress in rat liver. Exp. Toxicol. Pathol. 2011, 63, 433-441.

29. Shahrzad, S.; Aoyagi, K.; Winter, A.; Koyama, A.; Bitsch, I. Pharmacokinetics of gallic acid and its relative bioavailability from tea in healthy humans. J. Nutr. 2001, 131, 1207-1210.

30. Emmons, C.L.; Peterson, D.M. Antioxidant activity and phenolic content of oatas affected by cultivar and location. Crop Sci. 2001, 41, 1676-1681.

31. Luke, R.; Howard, J.R. Antioxidant capacity and phenolic content in blue berries as affected by genotype and growing season. J. Sci. Food Agric. 2003, 83, 1238-1247.

32. Hong, Y.J.; Tomas-Barberan, F.A.; Kader, A.; Mitchell, A.E. The flavonoid glycosides and procyanidin composition of Deglet Noor dates (Phoenix dactylifera). J. Agric. Food Chem. 2006, $54,2405-2411$.

33. Sanchez-Moreno, C. Review: Methods used to evaluate the free radical scavenging activity in foods and biological systems. Food Sci. Technol. Int. 2002, 8, 121-137.

34. Chaira, N.; Smaali, M.I.; Martinez-Tome, M.; Mrabet, A.; Murcia, M.A.; Ferchichi, A. Simple phenolic composition, flavonoid contents and antioxidant capacities in water-methanol extracts of Tunisian common date cultivars (Phoenix dactylifera L.). Int. J. Food Sci. Nutr. 2009, 60, 316-329.

35. Bilgari, F.; Alkarkhi, A.F.M.; Easa, A.M. Antioxidant activity and phenolic content of various date palm (Phoenix dactylifera) fruits from Iran. Food Chem. 2008, 107, 1636-1641.

36. Wuytac, T.; AbdElgawad, H.; Staelens, J.; Asard H.; Boeckx, P.; Verheyen, K.; Samson, R. The response of the foliar antioxidant system and stable isotopes $(\delta 13 \mathrm{C}$ and $\delta 15 \mathrm{~N})$ of white willow to low-level air pollution. Plant Physiol. Biochem. 2013, 67, 154-161,

37. Shinmoto, H.; Dosako, S.; Nakajima, I. Antioxidant activity of bovine lactoferrin on iron/ascorbate induce lipid peroxidation. Biosci. Biotechnol. Biochem. 1992, 56, 2079-2080.

38. Bilgari, F.; Alkarkhi, A.F.M.; Easa, A.M. Cluster analysis of antioxidant compounds in dates (Phoenix dactylifera): Effect of long-term cold storage. Food Chem. 2009, 112, 998-1001.

39. Cushnie, T.P.T.; Lamb, A.J. Antimicrobial activity of flavonoids. Int. J. Antimicrob. Agents 2005, 26, 343-356.

40. Nasir, M.U.; Hussain, S.; Jabbar, S.; Rashid, F.; Khalid, N.; Mehmood, A. A review on the nutritional content, functional properties and medicinal potential of dates. Sci. Lett. 2015, 3, 17-22.

41. Johnson, D.V.; Al-Khayri, J.M.; Jain, S.M. Introduction: Date production status and prospects in Asia and Europe. In Date Palm Genetic Resources and Utilization. 2: Asia and Europe; Al-Khayri, J.M., Jain, S.M., Johnson, D.V., Eds.; Springer: Dordrecht, The Netherlands, 2015; Volume 2, pp. 1-16.

42. Shi, L.E.; Zheng, W.; Aleid, S.M.; Tang, Z.X. Date pits: Chemical composition, nutritional and medicinal value, utilization. Crop Sci. 2014, 4, 1322-1330.

43. Borochov-Neori, H.; Judeinstein, S.; Greenberg, A.; Volkova, N.; Rosenblat, M.; Aviram, M. Antioxidant and antiatherogenic properties of phenolic acid and flavonol fractions of fruits of "Amari" and "Hallawi" date (Phoenix dactylifera L.) Varieties. J. Agric. Food Chem. 2015, 63, 3189-3195. 
44. Taha, K.K.; Al Ghtani, F.M. Determination of the elemental contents of date palm (Phoenix dactylifera L.) from Kharj Saudi Arabia. World Sci. News 2015, 6, 125-135.

45. Bouaziz, A.M.; Besbes, S.; Blecker, C.; Wathelet, B.; Deroanne, C.; Attia, H. Protein and amino acid profiles of Tunisian Deglet Nour and Allig date palm fruit seeds. Fruits 2008, 63, 37-43.

46. Salim, S.; Ahmed, A. Protein and amino acid contents of some Saudi Arabian date palm seeds (Phoenix dactylifera L.). Arab. Gulf. J. Sci. Res. 1992, 10, 1-9.

47. Shinwari, M.A. Date palm. In Encyclopaedia of Food Science, Food Technology \& Nutrition; Macrae, R., Robinson, R.K., Sadler, M.J., Eds.; Academic Press: London, UK, 1993; Volume 2, pp. 1300-1305.

48. Rinderknecht, $\mathrm{H}$. The free amino acid of dates in relation to their darkening maturation and storage. Food Res. 1959, 24, 298-304.

49. Ahmed, I.A.; Ahmed, A.W.K.; Robinson, R.K. Chemical composition of date varieties as influenced by the stage of ripening. Food Chem. 1995, 54, 305-309.

50. Messaoudi, R.S.; Abbeddou, S.; Mansouri, A.; Calokerinos, A.C.; Kefalas, P. Phenolic profile and antioxidant activity of date-pits of seven algerian date palm fruit varieties. Int. J. Food Prop. 2013, 16, 1037-1047.

51. Al-Farsi, M.A.; Lee, C.Y. Optimization of phenolics and dietary fibre extraction from date seeds. Food Chem. 2008, 108, 977-985.

52. Lobo, V.; Patil, A.; Phatak, A.; Chandra, N. Free radicals, antioxidants and functional foods: Impact on human health. Pharmacogn. Rev. 2010, 4, 118-126.

53. Traber, M.G. Vitamin E. In Modern Nutrition in Health and Disease, 10th ed.; Shils, M.E., Shike, M., Ross, A.C., Caballero, B., Cousins, R., Eds.; Lippincott Williams \& Wilkins: Baltimore, MD, USA, 2006; pp. 396-411.

54. Gill, S.; Tuteja, N. Reactive oxygen species and antioxidant machinery in abiotic stress tolerance in crop plants. Plant Physiol. Biochem. 2010, 48, 909-930.

55. Assirey, E.A. Nutritional composition of fruit of 10 date palm (Phoenix dactylifera L.) cultivars grown in Saudi Arabia. J. Taibah Univ. Sci. 2015, 9, 75-79.

56. Al-Hooti, S.; Sidhu, J.S.; Qabazard, H. Studies on the physico-chemical characteristics of date fruits of five UAE cultivars at different stages of maturity. Arab. Gulf. J. Sci. Res. 1995, 13, 553-569.

57. Al-Hooti, S.; Sidhu, J.S.; Qabazard, H. Physicochemical characteristics of five date fruit cultivars grown in the United Arab Emirates. Plant Foods Hum. Nutr. 1997, 50, 101-113.

58. Mohamed, R.M.A.; Fageer, A.S.M.; Eltayeb, M.M.; Ahmed, I.A.M. Chemical composition, antioxidant capacity, and mineral extractability of Sudanese date palm (Phoenix dactylifera L.) fruits. Food Sci. Nutr. 2014, 2, 478-489.

59. Appel, L.J.; Moore, T.J.; Obarzanek, E.W.; Vollmer, M.; Svetkey, L.P.; Sacks, F.M.; Bray, G.A.; Vogt, T.M.; Cutler, J.A.; Windhauser, M.M.; et al. A clinical trial of the effects of dietary patterns on blood pressure. N. Engl. J. Med. 1997, 336, 1117-1124.

60. Gasim, A.A. Changes in sugar quality and mineral elements during fruit development in five date palm cultivars in Al-Madinah Al-Munawwarah, JKAU. Science 1994, 6, 29-36.

61. Fahad, A.L.; Juhaimi, K.G.; Özcan, M.M. Physicochemical properties and mineral contents of seven different date fruit (Phoenix dactylifera L.) varieties growing in Saudi Arabia. Environ. Monit. Assess. 2014, 186, 2165-2170. 
62. Cheung, L.M.; Cheung, P.C.K.; Ooi, V.E.C. Antioxidant activity and total phenolics of edible mushroom extracts. Food Chem. 2003, 7, 249-255.

63. Patro, B.S.; Rele, S.; Chintalwar, G.J.; Chattopadhyay, S.; Adhikari, S.; Mukherjee, T. Protective activities of some phenolic 1,3-diketones against lipid peroxidation: Possible involvement of the 1,3-diketone moiety. Chem. Biochem. 2002, 3, 364-370.

64. Sinhaa, A.K.; Giblena, T.; AbdElgawad, H.; de Rop, M.; Asard, H.; Blust, R.; Boeck, G. Regulation of amino acid metabolism as a defensive strategy in the brain of three freshwater teleosts in response to high environmental ammonia exposure. Aquat. Toxicol. 2013, 130, 86-96.

65. Alasalvar, C.; Shahidi, F.; Liyanapathirana, C.M.; Ohshima, T. Turkish tombul hazelnut (Corylus avellana L.). 1. Compositional characteristics. J. Agric. Food Chem. 2003, 51, 3790-3796.

66. Gomaa, N.H.; AbdElgawad, H.R. Phytotoxic effects of Echinochloa colona (L.) Link. (Poaceae) extracts on the germination and seedling growth of weeds. Span. J. Agric. Res. 2012, 10, 492-501.

67. Potters, G.; Horemans, N.; Bellone, S.; Caubergs, R.J.; Trost, P.; Guisez, Y.; Asard, H. Dehydroascorbate influences the plant cell cycle through a glutathione-independent reduction mechanism. Plant Physiol. 2004, 134, 1479-1487.

68. Siebert, K.J. Modeling the flavor thresholds of organic acids in beer as a function of their molecular properties. Food Qual. Pref. 1999, 10, 129-137.

69. Agusa, T.; Kunito, T.; Yasunaga, G.; Iwata H.; Subramanian A.; Ismail, A.; Tanabe, S. Concentrations of trace elements in marine fish and its risk assessment in Malaysia. Mar. Pollut. Bull. 2005, 51, 896-911.

Sample Availability: Not available.

(C) 2015 by the authors; licensee MDPI, Basel, Switzerland. This article is an open access article distributed under the terms and conditions of the Creative Commons Attribution license (http://creativecommons.org/licenses/by/4.0/). 